\title{
ARTIGO 23
}

\section{AUTOFIX: UMA TECNOLOGIA PARA FIXAÇÃO SEGURA DO TUBO OROTRAQUEAL}

Lidiane Marha de Souza Oliveira ${ }^{1}$

Ana Carine Goersch Silva ${ }^{1}$

Aglauvanir Soares Barbosa ${ }^{2}$

Anna Paula Sousa da Silva ${ }^{3}$

Islene Victor Barbosa ${ }^{1}$

Rita Monica Borges Studart ${ }^{1}$ http://orcid.org/0000-0003-2004-6180

http://orcid.org/0000-0001-8067-6120

https://orcid.org/0000-0003-4909-563X

http://orcid.org/0000-0002-8833-6881

http://orcid.org/0000-0003-3523-7238

http://orcid.org/0000-0002-5862-5244

Objetivo: Construir algoritmos de intervenção de enfermagem pré-hospitalar para vítimas de trauma. Metodologia: Revisão Narrativa da Literatura, entre 2008 e 2019, nas principais bases de dados. Dois revisores independentes realizaram a avaliação crítica, extração e síntese dos dados. A construção dos algoritmos resultou do processo interpretativo da revisão narrativa por três peritos na área. Utilizou-se o modelo teórico de Virgínia Henderson. Resultados: Obtiveram-se 17 documentos, seis foram incluídos no desenvolvimento dos metaparadigmas Saúde, Pessoa e Ambiente e 16 na elaboração e construção de Algoritmos de avaliação, diagnóstico e intervenções de enfermagem às vítimas de trauma. Conclusões: A revisão possibilitou a operacionalização do modelo teórico de Henderson para a assistência pré-hospitalar permitindo a criação de algoritmos orientadores da prática de enfermagem.

Descritores: Enfermagem Baseada em Evidências; Teoria de Enfermagem; Trauma; Assistência Pré-Hospitalar; Assistência de Enfermagem.

\section{PRE-HOSPITAL NURSING INTERVENTIONS: NARRATIVE REVIEW}

Objective: To construct pre-hospital nursing intervention algorithms for trauma victims.

Methodology: Literature Narrative Review, between 2008 and 2019, in the main databases. Two independent reviewers carried out the critical evaluation, extraction and synthesis of data. The construction of the algorithms resulted from the interpretive process of the narrative review by three experts. The theoretical model of Virginia Henderson was used. Results: Seventeen documents were obtained, six were included in the development of the metaparadigm Health, Person and Environment and 16 were included in the development and construction of assessment, diagnosis and nursing interventions algorithms for trauma victims. Conclusions: The review enabled the operationalization of Henderson's theoretical model for pre-hospital care, allowing the creation of algorithms to guide nursing practice.

Descriptors: Evidence-based Nursing; Nursing Theory; Trauma; Prehospital Care; Nursing Care.

\section{INTERVENCIONES DE ENFERMERÍA PREHOSPITALARIA: REVISIÓN NARRATIVA}

Objetivo: Construir algoritmos de intervención de enfermería prehospitalaria para víctimas de traumatismos. Metodología: Revisión narrativa de literatura, entre 2008 y 2019, en las principales bases de datos. Dos revisores independientes realizaron la evaluación crítica, extracción y síntesis de los datos. La construcción de los algoritmos fue el resultado del proceso interpretativo de la revisión por parte de tres expertos. Se utilizó el modelo de Virginia Henderson. Resultados: Se obtuvieron diecisiete documentos, seis se incluyeron en el desarrollo de los metaparadigmas y 16 se incluyeron en el desarrollo y construcción de algoritmos de evaluación, diagnóstico e intervenciones de enfermería. Conclusiones: La revisión permitió la operacionalización del modelo de Henderson para la atención prehospitalaria, permitiendo la creación de algoritmos.

Descriptores: Enfermería Basada en la Evidencia; Teoría de Enfermería; Trauma; Atención Prehospitalaria; Atención de Enfermería. 


\section{INTRODUÇÃO}

Dentre o acervo tecnológico comumente usados pelo paciente crítico, cita-se o suporte ventilatório mediante a ventilação mecânica (VM) com o uso de Tubo orotraqueal (TOT) ou traqueóstomo para assegurar uma via aérea. Estes dispositivos são fundamentais para as trocas gasosas e equilibrio entre demanda e oferta de oxigênio(1). A VM nesse contexto, substitui total ou parcial, a respiração espontânea e está indicada na insuficiência respiratória aguda ou crônica agudizada, sendo imprescindivel os cuidados de enfermagem.

Os cuidados de enfermagem ao paciente em uso de VM não se restringem somente à administração dos medicamentos prescritos e percepção do agravamento do seu quadro, envolve também cuidados específicos como a fixação do TOT, aspiração traqueal e de vias aéreas superiores, controle da pressão do balonete, observação da sincronia entre o paciente e a máquina e monitorização dos parâmetros ventilatórios ${ }^{(2)}$

A fixação segura do TOT deve ser realizada de forma sistemática e com bastante cautela, em virtude de ser fundamental para a segurança, conforto e confiabilidade do procedimento, aliado ao fato de contribuir para manter uma via aérea artificial pérvia. Durante a fixação do TOT, alguns cuidados devem ser observados, incluindo a periodicidade de troca para evitar a mobilização do tubo e permitir maior controle da localização adequada(3)

O desenvolvimento de uma tecnologia voltada para a fixação segura tem sua importância respaldada na política de segurança do paciente, visto ser uma das principais metas dos serviços de saúde, tanto em decorrência do impacto com os gastos resultantes da falta de segurança, quanto em virtude de danos sofridos durante o cuidado prestado aos pacientes ${ }^{(4)}$

A escolha de pesquisar sobre o assunto ocorreu em virtude de trabalhar em UTI e perceber a complexidade que envolve a assistência desses pacientes, ocorrendo algumas vezes a extubação acidental. Diante disso foi desenvolvida, uma forma de fixação segura que foi denominada de autofix.

Sendo assim, o estudo trará contribuições relevantes para a Enfermagem e toda equipe multiprofissional, acerca do procedimento de fixação segura do tubo orotraqueal. Diante disso, o objetivo do estudo foi construir e validar uma tecnologia diferenciada para fixação do tubo orotraqueal em pacientes críticos.

\section{METODOLOGIA}

\section{Tipo de Estudo}

Trata-se de um estudo descritivo metodológico para construção e validação de um protótipo para fixação segura denominado "autofix" utilizando uma ferramenta tecnológica para promoção da saúde dos pacientes intubados.

\section{Participantes da Pesquisa}

Participaram da pesquisa 15 juízes especialistas profissionais da saúde, equipe multiprofissional, médicos intensivistas, enfermeiros especialistas e fisioterapeutas intensivistas. Para a seleção dos juízes-especialistas foram estabelecidos como critérios: a titulação, participação de eventos, produção cientifica e o tempo de atuação com a temática em discussão. Fez parte deste comitê apenas os juízes que obtiveram, no mínimo, pontuação igual ou superior a cinco pontos a partir dos seguintes critérios apresentados no quadro 1 .

Quadro 1 - Critérios de seleção para os juizes - especialistas da área da saúde. Fortaleza, Ceará. Brasil, 2019.

\begin{tabular}{|l|l|}
\hline CRITÉRIOS & PONTOS \\
\hline Ser doutor na área de interesse & 4 pontos \\
\hline Ser mestre na área de interesse & 3 pontos \\
\hline Ser especialista na área de interesse & 2 pontos \\
\hline Ter no mínimo cinco anos de experiência na área de saúde & 3 pontos \\
\hline Ter produção científica & 2 pontos \\
\hline Ter participado em eventos da área de saúde nos últimos 5 anos & 1 pontos \\
\hline
\end{tabular}

Os juizes foram selecionados através da técnica de amostragem não probabilística intencional, pois possibilitou ao pesquisador selecionar os juízes por decisão própria, baseado na especialidade de cada um acerca do tema estudado(5).

\section{Etapas da Pesquisa}

O desenvolvimento da pesquisa para construção do artefato tecnológico ocorreu em quatro fases: 1a fase: diagnóstico situacional e estudo bibliográfico com a finalidade de embasamento teórico; 2a ${ }^{\mathrm{a}}$ fase: criação da ferramenta tecnológica, concepção e desenvolvimento do artefato tecnológico; 3a fase: avaliação do protótipo por juízes especialistas e; 4 a fase: compilação e análise de dados. A pesquisa teve início em março de 2018 com o levantamento bibliográfico, na sequência, entre julho e agosto foi dado largada a criação do protótipo da tecnologia e a terceira parte. ocorreu já em 2019, durante o mês de abril com a validação dos juízes avaliando a tecnologia.

Inicialmente foi realizado um levantamento bibliográfico, das principais publicações acerca da segurança do paciente, extubação acidental, humanização e promoção da saúde, tecnologia da saúde e de enfermagem e cuidados de enfermagem ao paciente em ventilação mecânica, com o intuito de levantar as informações existentes para compor o artefato tecnológico.

Em seguida ocorreu a elaboração e desenvolvimento do artefato tecnológico e incorporação das hipóteses do estudo como solução do problema identificado em um ambiente controlado. A tecnologia elaborada foi nomeada por "AUTOFIX" que se caracteriza em um modelo de fixação 
para o tubo orotraqueal. Foi utilizado um programa em 3D denominado Blender. A finalidade foi proporcionar um protótipo mais próximo da realidade.

O autofix é composto por uma fita elástica dupla e macia, de fixação segura que perpassa por trás da cabeça na região occipital, proporcionando conforto e segurança para os pacientes entubados, bem como para os profissionais que prestam assistência a esses pacientes. A fita de fixação faz uma fixação dupla e centralizada do tubo, evitando assim o surgimento de ferimentos nos cantos da boca dos pacientes.

A fase laboratorial de desenvolvimento do artefato tecnológico ocorreu no Laboratório de Inovação do Núcleo de Aplicação em Tecnologia da Informação (NATI) da Universidade de Fortaleza (UNIFOR).

Para a validação do Protótipo, ocorreu a avaliação do artefato tecnológico por juizes selecionados com expertise na área.

\section{Coleta de Dados}

A coleta de dados na primeira fase ocorreu através de busca na literatura de artigos que falassem sobre o processo de suporte ventilatório em pacientes críticos e os riscos de lesões mucosas, nas seguintes bases de dados: Cinahl, Scielo e BVS; A coleta de dados na segunda fase foi uma das mais complicadas, devido o processo de criação da tecnologia, as adaptações e modificações que iam sendo necessárias realizar para melhoria do artefato tecnológico.

$\mathrm{Na}$ terceira etapa foi elaborado um instrumento estruturado direcionado aos juízes, contendo participante, profissão, tempo e área de atuação, titulação. Este questionário foi organizado conforme a escala de Likert, com itens distribuídos em três blocos, contendo perguntas referentes à avaliação do conteúdo do simulador.

\section{Análise de Dados}

Como forma de determinar o nivel de concordância entre os juízes especialistas foi calculado o Índice de Validade de Conteúdo (IVC)

Visando a objetividade da validade de conteúdo, calculouse o Índice de Validade de Conteúdo (IVC), considerando excelente o IVC dos itens (IVCi) maior ou igual a 0,78 e, média de IVC total de 0,90 ou superior. Adotou-se o seguinte padrão de avaliação: IVCi $\geq 0,78$ excelente, IVCi entre 0,60 e 0,71 bom, e IVC $i<0,59$ ruim $^{(5)}$.

Para a análise dos itens de avaliação da tecnologia criada, foi realizado o teste exato de distribuição binomial, indicado para pequenas amostras, sendo considerados nivel de significância p>0,05 e proporção de 0,95 de concordância para estimar a confiabilidade estatística dos IVC.

\section{Aspectos Éticos}

Os aspectos éticos foram respeitados em todas as etapas do desenvolvimento da pesquisa, respeitando assim os preceitos da Resolução 466/12(6). O projeto foi encaminhado ao Comitê de Ética em Pesquisa e recebeu parecer favorável com número: 2.435.893. Os juízes que participaram da validação assinaram Termo de Consentimento Livre e Esclarecido (TCLE) concordando em participar da pesquisa.

\section{RESULTADOS}

No total, 15 juízes, profissionais da saúde, responderam ao instrumento de avaliação do Autofix. No que tange à caracterização dos juízes, observou-se que a maioria era do sexo feminino (80\%). A idade mínima foi de 26 anos e a máxima de 49 anos, com média de 34,5 ^ 5,3 anos; a faixa etária de 30 a 39 anos foi a mais prevalente (80\%). Os juizes que participaram do processo de validação de conteúdo da tecnologia tinham profissões variadas: dez (66,7\%) eram enfermeiros, dois $(13,3 \%)$ eram médicos, dois (13,3\%) eram dentistas e um $(6,7 \%)$ era fisioterapeuta.

Constatou-se que todos os juízes eram especialistas $(100 \%)$, sete deles eram mestres $(46,7 \%)$ e um era doutor (93,3\%), com tempo de experiência assistencial de até 10 anos (73,3\%), sendo o tempo mínimo de três anos e máximo de 24 anos, e média de $9 \pm 5$ anos

Após a apresentação do protótipo (Figura 1) para os profissionais, seguindo as recomendações e critérios definidos para o julgamento, foi explicado que o mesmo deveria preencher uma ficha de avaliação do produto. elaborada pela autora.

Figura 1: Autofix no programa blender 3D. Fortaleza, Ceará Brasil, 2019.
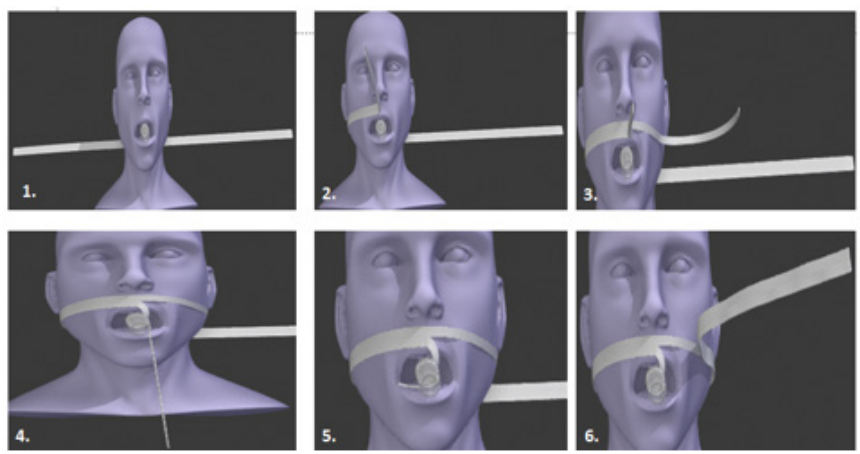

A ficha de avaliação foi composta por dados profissionais e institucionais e um descritivo acerca do artefato, com informações técnicas de usabilidade, seguido de questões fechadas dicotômicas ( $\operatorname{sim}$ ou não) e uma questão aberta sobre sugestões.

As questões dicotômicas referiam-se à segurança, facilidades quanto à colocação, higienização oral e 
movimentação do tubo, considerando a possibilidade do deslizamento do tubo.

Tabela 1. Índice de Validade de Conteúdo (IVC) e Teste Binomial dos itens de avaliação da tecnologia construída. Fortaleza, Ceará. Brasil, 2019.

\begin{tabular}{|c|c|c|c|}
\hline & Itens & IVCia & $\mathbf{p}_{n}^{\mathbf{b}}$ \\
\hline 1) & O Autofix é de fácil instalação? & 1,00 & 0,463 \\
\hline 2) & $\begin{array}{l}\text { Esse modelo de fixação poderá proporcionar uma maior segurança } \\
\text { na estabilidade do tubo orotraqueal durante os ciclos de } \\
\text { reanimação cardiopulmonar em uma situação de parada } \\
\text { cardiorrespiratória? }\end{array}$ & 1,00 & 0,463 \\
\hline 3) & $\begin{array}{l}\text { O Autofix trará mais segurança à fixação do tubo durante o } \\
\text { transporte do paciente de uma maca para o leito ou mesmo para a } \\
\text { mesa de exame de imagem ou cirúrgica? }\end{array}$ & 1,00 & 0,463 \\
\hline 4) & $\begin{array}{l}\text { Com esse modelo de fixação há maior segurança durante a } \\
\text { mudança de posição do paciente no leito e do ventilador } \\
\text { mecânico? }\end{array}$ & 1,00 & 0,463 \\
\hline 5) & $\begin{array}{l}\text { Há maior praticidade em relação à aspiração e à limpeza da } \\
\text { cavidade oral? }\end{array}$ & 0,93 & 0,537 \\
\hline 6) & $\begin{array}{l}\text { O Autofix proporcionaria maior facilidade para a abordagem } \\
\text { odontológica do paciente? }\end{array}$ & 0,93 & 0,537 \\
\hline 7) & $\begin{array}{l}\text { Poderia haver maior durabilidade desse modelo de fixação mesmo } \\
\text { quando exposto à umidade e à secreção? }\end{array}$ & 0,87 & 0,171 \\
\hline 8) & $\begin{array}{l}\text { Com esse modelo de fixação poderá existir menor risco de } \\
\text { extubação acidental? }\end{array}$ & 1,00 & 0,463 \\
\hline 9) & O Autofix traria beneficio à estética visual do paciente entubado? & 0,87 & 0,171 \\
\hline 10) & $\begin{array}{l}\text { Esse modelo de fixação traria maior estabilidade do tudo } \\
\text { orotraqueal durante a manobra de pronação no leito usada para } \\
\text { otimizar a ventilação mecânica em determinados casos? }\end{array}$ & 1,00 & 0,463 \\
\hline
\end{tabular}

Como exposto na tabela acima, nos itens avaliados pelos juízes, observa-se o predomínio da concordância entre os juizes em relação a instalação do Autofix, sua fixação com segurança, bem como a diminuição do risco de extubação acidental do tubo e pôr fim a concordância foi sobre a maior estabilidade do tubo com esse tipo de fixação, todos com IVC de 1,00 .

\section{DISCUSSÃO}

O ambiente de UTI é complexo, com muita tecnologia e aparelhos que ajudam a salvar vidas, dentro desse contexto o profissional de saúde desempenha um papel fundamental na assistência prestada ao paciente e à sua família durante o período de internação do seu familiar no ambiente de terapia intensiva, sendo um momento muito delicado, de medo, dúvidas e frustações para todos os envolvidos ao se depararem com seu familiar ligados a muitos aparelhos e fazendo uso de $\mathrm{VM}^{(7)}$

A VM é de grande importância na UTI devido à necessidade de promover um adequado aporte ventilatório e, por tal, é denominado um dos suportes à vida de maior relevância e um dos recursos mais utilizados para manutenção do padrão respiratório de pacientes em que o organismo é incapaz de manter o ciclo respiratório sem a ajuda do suporte ventilatório $^{(8)}$.

As Unidades de Terapia Intensiva (UTI), dispõem de um arsenal tecnológico, com materiais de alta complexidade, com o propósito de atender pacientes graves, mas também é um local onde se observa a ocorrência de eventos adversos $(\mathrm{EA})^{(9)}$.
A promoção de uma assistência à saúde segura e livre de danos desnecessários dá-se através de profissionais capacitados no uso das tecnologias como também no que se refere à segurança do paciente (SP). Nesse sentido, evidenciase a importância de incentivar e valorizar o ensino da segurança durante o processo de formação dos profissionais da saúde ${ }^{(10)}$.

A Organização Mundial da Saúde (OMS) criou em 2011 um guia com diretrizes para o ensino multiprofissional da SP, o Patient Safety Curriculum Guide: Multiprofessional Edition. Essas diretrizes objetivam promover e facilitar, em âmbito mundial, o ensino da SP com o propósito de reduzir os EA e promover uma assistência à saúde com qualidade(11). Outro problema recorrente relacionado a VM são as extubações acidentais, que geram complicações e podem piorar o quadro do paciente gravemente enfermo.

As complicações da extubação acidental são maiores quando a retirada do dispositivo ventilatório ocorre em pacientes com estímulo respiratório reduzido, em virtude de lesões neurológicas, por sedação ou coma, pois leva a retenção de gás carbônico (CO2), redução de oxigênio $(\mathrm{O} 2)$ e a necessidade de reintubação, fato esse que pode contribuir para uma piora do quadro clínico do paciente $e^{(12)}$

A extubação acidental pode ocorrer devido à agitação psicomotora do paciente, falta de sedação adequada, inadequada fixação do dispositivo ventilatório, cuff do tubo orotraqueal furado ou vazio, tração ou peso excessivo dos acessórios do ventilador mecânico, bem como, consequente ao manuseio inadequado do paciente pela equipe multiprofissional, caracterizando, assim, um evento adverso do cuidado.

A tecnologia em saúde é apontada como um fenômeno complexo que gera reflexões e conversações cotidianas sobre as diversas experiências de cuidado ao cliente que dela depende e ainda implicações nos modos de agir específicos no cuidado de enfermagem. A tecnologia mostrase desconhecida e impossibilita o cuidado apropriado caso não ocorra o processo de familiarização. Destacando que é primordial conhecer a linguagem dos equipamentos tecnológicos ${ }^{(13)}$

As tecnologias de enfermagem atuam de modo a fortalecer e qualificar o cuidado, permitindo a tomada de decisão mediante o raciocínio clínico, levando em consideração a individualização do indivíduo. Assim, é importante que os enfermeiros se adaptem a esse contexto de avanços, buscando qualificação e aperfeiçoamento da prática, sem esquecer que é o cuidado quem utiliza a tecnologia em direção a um cuidado de enfermagem mais eficaz e seguro.

Os processos assistenciais no auxílio durante os cuidados aos pacientes críticos em uso de dispositivos invasivos como suporte ventilatório, devem estar centrados na redução de riscos de vida, bem como na preservação da mucosa integra 
livre de lesões cutâneo-mucosas. Dessa forma pode-se garantir a manutenção da via respiratória, evitando possiveis danos temporários ou permanentes e períodos logos de internações.

As lesões cutâneo-mucosas ocasionadas pelo uso de tuへbo orotraqueal dependem de vários fatores, como as condições clínicas do paciente, permanência proᄀlongada dos dispositivos e cuidados inadequados dispensa $\neg$ dos pela equipe de Enfermagem. Ressalta-se a relevância da capacitação da equipe de Enfermagem para atuar nos cuidados que envolvem o paciente com dispositivo invasivo de via aérea.

Asegurança para a prevenção de lesões cutâneo-mucosas em pacientes submetidos a dispositivos invasivos em vias aéreas inferiores, observa-se pouca produção científica na Enfermagem e áreas afins. É necessário a importância de resgatar esse enfoque, considerando que essas lesões estão diretamente relacionadas aos cuidados de Enfermagem, podendo ser prevenidas a partir da inclusão de ações que garantam a segurança do paciente.

Sendo assim o uso de um dispositivo diferenciado que proporcione segurança ao paciente entubado, que facilite o manuseio, a movimentação do tubo e a higienização oral, para prevenir tais complicações será de grande ajuda e melhoria na qualidade da assistência prestada a esses pacientes graves.

O autofix, será um instrumento diferenciado que irá proporcionar segurança e melhorias ao paciente em uso de VM, onde o mesmo irá auxiliar na diminuição das extubações e na diminuição do risco de surgimento de lesões na mucosa. Ele é diferenciado pela forma de fixação do tubo a cavidade oral do paciente, bem como pelo material utilizado na sua confecção.

\section{Limitações do Estudo}

Acreditamos que a limitação do estudo tenha sido a sua execução só na Unidade Especializada, e o que pode não evidenciar a realidade de outras instituições de saúde.

\section{Contribuição do estudo para a prática}

O estudo amplia a área de conhecimento de enfermagem e pode se constituir em um sinalizador para estudos metodológicos.

\section{CONCLUSÃO}

Foi construído e validado uma tecnologia diferenciada denominada autofix para fixação de tubo orotraqueal em pacientes críticos. Foi desenvolvida para atuar como estratégia de segurança e humanização do paciente crítico em uso da ventilação mecânica.

A validação do conteúdo teve excelente IVC global $(0,97)$ devendo-se assim ser considerado no contexto hospitalar como um instrumento capaz de favorecer o paciente e equipe para uma assistência humanizada e qualificada. Diante do exposto, acredita-se que os objetivos deste estudo foram plenamente atingidos com o desenvolvimento do autofix e sua avaliação positiva pelos especialistas.

\section{Contribuição dos autores}

Concepção e/ou desenho, análise e interpretação dos dados, redação do artigo, revisão crítica, revisão final:Lidiane Marha de Souza Oliveira, Ana Carine Goersch Silva, Aglauvanir Soares Barbosa, Anna Paula Sousa da Silva, Islene Victor Barbosa, Rita Monica Borges Studart. 


\section{REFERÊNCIAS}

1. Loss SH, Oliveira RP, Maccari JG, Savi A, Boniatti MM, Hetzel MP, et al. A realidade dos pacientes que necessitam de ventilação mecânica prolongada: um estudo multicêntrico. Rev. bras. ter. intensiva [internet]. 2015 [cited 2109 jun 16];27(1):26-35. Available from: http:// www.scielo.br/pdf/rbti/v27nl/0103-507X-rbti-27-01-0026.pdf

2. Melo EM, Teixeira CS, Oliveira RT, Almeida DT, Veras JEGLF, Frota NM, Studart RMB. Cuidados de enfermagem ao utente sob ventilação mecânica internado em unidade de terapia intensiva. Rev.. Enf. Ref. [internet] 2014. [cited 2019 jun 16]:4(1):55-63. Available from: http://www.scielo.mec.pt/scielo.php?script=sci_ arttextEpid=S087402832014000100007\&lng=ptEnrm=i\&tlng=pt

3. Pinto DM de, Schons E dos S, Busanello J, Costa VZ da. Patient safety and the prevention of skin and mucosal lesions associated with airway invasive devices. Rev Esc Enferm USP [internet]. 2015 [cited 2019 jun 16]:49(5):775-782. Available from: http://www.scielo.br/pdf/ reeusp/v49n5/pt_0080-6234-reeusp-49-05-0775.pdf

4. Françolin L, Gabriel CS, Bernardes A, Silva AEBC, Brito MFP. Machado JP. Gerenciamento da segurança do paciente sob a ótica dos enfermeiros. Rev Esc Enferm USP [internet]. 2015 [cited 2019 jun 16];49(2):277-83. Available from: http://www.scielo.br/pdf/reeusp/ v49n2/pt_0080-6234-reeusp-49-02-0277.pdf

5. Polit DF, Beck CT. Fundamentos de pesquisa em enfermagem: avaliação de evidências para a prática da enfermagem. 7. ed. Porto Alegre: Artmed, 2011. 670p.

6. Ministério da Saúde (BR). Conselho Nacional de Saúde. Resolução no 466 de 12 de dezembro de 2012. Brasilia (DF): Ministério da Saúde. 2013. Available from: http://bvsms.saude.gov.br/bvs/saudelegis/cns/2013/ res0466_12_12_2012.html.

7. Cruz EJER, Souza NVDO, Correa RA, Pires AS. Dialética de sentimentos do enfermeiro intensivista sobre o trabalho na terapia intensiva. Escola Anna Nery [internet]. 2014 [cited 2019 jun 18];18(3):47985. Available from: http://www.scielo.br/scielo.php?script=sci_ arttextEpid=S1414-81452014000300479.
8. Hinkle JL, Cheever KH. Brunner \& Suddarth tratado de enfermagem médico-cirúrgica. 13.ed. Rio de Janeiro-RJ: Guanabara Koogan, 2016.

9. Lima CSP. Barbosa SFF. Ocorrência de eventos adversos como indicadores de qualidade assistencial em unidade de terapia intensiva. Rev Enferm UERJ [internet]. 2015 [cited 2019 jun 18]:23(2):221-8. Available from: http://www.facenf.uerj.br/v23n2/v23n2al3.pdf

10. Da Silva JA, Pinto FCM. Avaliando o Impacto da Estratégia de Segurança do Paciente Implantada em uma Unidade de Clínica Médica de um Hospital Universitário sob a Perspectiva da Dimensão da Atenção à Saúde. Rev. Adm. Saúde [internet]. 2017 [cited 2019 jun 18];17(66). Available from: http://cqh.org.br/ojs-2.4.8/index.php/ras/ article/view/10/20

11. World Health Organization. Segurança do Paciente em Serviços de Saúde Limpeza e Desinfecção de superficies [internet]. 2011 [cited 2019 jun 18]; Available from: http://iris.paho.org/xmlui/ handle/123456789/3582

12. Beccaria LM, Tavares TG, Penascho MCB, Faria JIL, Jabur MRL, Oliveira KAD. Extubação acidental e dano causado ao paciente em um hospital de ensino. CuidArte Enferm [internet]. 2018 [cited 2019 jun 18];12(1):11-17. Availabel from: http://www.webfipa.net/facfipa/ner/ sumarios/cuidarte/2018vl/1l.pdf

13. Silva RC, Ferreira MA. Tecnologia na terapia intensiva e suas influências nas ações do enfermeiro. Rev Esc Enferm USP [internet]. 2011 [cited 2019 jun 18]:45(6):1403-11. Available from: http://www.scielo. br/scielo.php?script=sci_arttextEpid=S0080-62342011000600018

14. Dornelles C, Oliveira GB, Schwonke CRGB, Silva JRS. Experiências de doentes críticos com a ventilação mecânica invasiva. Esc Anna Nery [internet]. 2012 [cited 2019 jun 18];16(4):796801. Available from: http://www.scielo.br/scielo.php?script=sci_ arttext\&pid=S1414-81452012000400022 